\title{
Relação entre gênero e desempenho neuropsicomotor de crianças em Belém, Brasil
}

\author{
Relationship between gender and psychomotor performance \\ of children in Belém, Brazil
}

Mariane Lopes da Silva ${ }^{1}$

Lilia Iêda Chaves Cavalcante ${ }^{1}$

Sabine Heumann ${ }^{1}$

Telma Vitorina Ribeiro Lima ${ }^{1}$

${ }^{1}$ Programa de Pós-

Graduação em

Teoria e Pesquisa do

Comportamento,

Universidade Federal do

Pará. R. Augusto Corrêa 1,

Guamá. 66075-110

Belém PA Brasil.

marianelopes.to@

hotmail.com

\begin{abstract}
This study aimed to analyze the neuropsychomotor performance of children by implementing the Denver Developmental Screening Test-II (DDST-II). We evaluated a sample of 318 children aged 36 to 48 months. Results indicated that girls performed better in three of the four areas analyzed in the test: Personal-Social $(p<0.001)$, Fine Motor-adaptive $(p=0.020)$ and Language ( $p=0.028$ ). No significant difference was observed between genders in the Gross Motor skills area. Analyzing the performance of children in implementing the tasks expected in the test, we found significant differences in the following items: in the Personal-Social area, the worst-performing item was "Brushes teeth without help"; in the Fine Motor area, the worst-performing item was "shows the longest line"; in the Language area, the worst-performing items were "Knows two adjectives", "Knows four actions" and "Understands four prepositions"; and in the Gross Motor area, the worst-performing item was "Hops on one foot". The results suggest that socially-imposed standards, based on gender differences, may interfere with the neuropsychomotor behavior of children. Furthermore, knowing such development profile is crucial in the formulation of public policies and actions that can contribute to child development.
\end{abstract}

Key words Child development, Development evaluation, Gender differences
Resumo Este estudo objetivou analisar o desempenho neuropsicomotor de crianças a partir da realização do Teste de Triagem de Desenvolvimento de Denver II (TTDD-II). Foram avaliadas 318 crianças, com idade entre 36 a 48 meses. Os resultados indicaram que as meninas obtiveram melhor desempenho em três das quatro áreas pesquisadas: Pessoal Social $(p<0,001)$, Motor Fino $(p=$ $0,020)$ e Linguagem ( $p=0,028)$. Na área Motor Amplo não foi observada diferença significativa entre os gêneros. Ao se analisar o desempenho das crianças na execução das tarefas previstas no teste, constatou-se diferença significativa nos seguintes itens: na área Pessoal Social, o item mais falho foi "escova os dentes sem ajuda"; na área Motor fino, o item "indica a linha mais longa" apresentou mais falhas; na área da Linguagem, os itens mais falhos foram "conhece 2 adjetivos", "conhece 4 ações" e "compreende 4 preposições"; na área Motor Amplo foi o item mais falho "pula num pé só”. A análise dos resultados aponta que padrões socialmente impostos, baseados em diferenças de gênero, podem interferir nos comportamentos neuropsicomotores de crianças. Além disso, conhecer tal perfil de desenvolvimento é de fundamental importância na formulação de políticas públicas e de ações capazes de contribuir para o desenvolvimento infantil.

Palavras-chave Desenvolvimento infantil, Avaliação do desenvolvimento, Diferenças de gênero 


\section{Introdução}

Compreender o processo de desenvolvimento humano tem sido uma preocupação mundial, particularmente entre os países com baixo crescimento econômico e elevados níveis de desigualdade social. Estudos realizados em países de baixa e média renda ressaltaram a importância da intervenção nos primeiros anos de vida como fator primordial para melhorar o neurodesenvolvimento de crianças ${ }^{1}$. O Desenvolvimento Neuropsicomotor (DNPM) se apresenta como um importante parâmetro de avaliação nos anos iniciais de vida, permitindo aos pesquisadores e profissionais detectarem precocemente eventuais alterações.

O DNPM está associado à maturação do sistema nervoso central (SNC) e compreende quatro grandes campos: Motor Amplo, Motor Fino (Adaptativo), Social e Linguístico. Contudo, as habilidades que compõem cada campo surgem em sequência: as habilidades mais simples servem de base paras as mais complexas, sendo importante compreendê-las e distingui-las. À medida que os reflexos primitivos vão sendo inibidos, a criança adquire novas habilidades dentro dos quatro campos citados, alcançando os marcos de desenvolvimento. Cada indivíduo possui seu próprio ritmo de crescimento, sendo esperado que não se distancie drasticamente do padrão observado para a sua idade, dentre outros critérios estabelecidos ${ }^{2}$.

A avaliação do DNPM permite a exploração dos marcos do desenvolvimento em populações infantis, devendo idealmente ser realizada por meio de instrumentos padronizados. O diagnóstico precoce de atraso do DNPM possibilita intervenções terapêuticas oportunas, reduzindo a incidência de complicações neurológicas muitas vezes permanentes. Estudo realizado em Curralinho, no Estado de Minas Gerais, confirma a importância desse diagnóstico por meio do Teste de Triagem do Desenvolvimento Denver II (TTDD II) nas consultas rotineiras de puericultura, podendo antever prejuízos nos pacientes e atuar preventivamente. Afirma ainda que a carência de acompanhamento qualificado pode levar a danos humanos e sociais, além do prejuízo financeiro ao município resultante da não atuação precoce em caso de atrasos no desenvolvimento que poderiam ter sido evitados ${ }^{2}$.

Vários testes e escalas vêm sendo utilizados para avaliar o desenvolvimento infantil no país, sendo a maioria originada em outros países, a exemplo da Escala de Bayle ${ }^{3}$ e da Escala Alberta ${ }^{4}$.
Existem, contudo, alguns instrumentos padronizados por pesquisadores brasileiros, como a Escala de Rosa Neto ${ }^{5}$. O TTDD II, apesar de ainda não validado, tem sido bastante utilizado em pesquisas realizadas em diferentes municípios brasileiros: Porto Alegre- $\mathrm{RS}^{6}$, Ribeirão Preto-SP7, São Carlos-SP ${ }^{8}$, São Paulo-SP ${ }^{9}$, Cuiabá-MT ${ }^{10}$, Belo Ipatinga-MG ${ }^{11}$, Belo Horizonte- $\mathrm{MG}^{12}$, entre outros. Possivelmente devido à sua rápida e fácil aplicabilidade por técnicos e pesquisadores de quaisquer áreas, desde que devidamente treinados. Destaca-se que a maioria dos estudos foi realizada no Centro-Sul do país, sendo que apenas dois em cidades do Norte do Brasil ${ }^{13,14}$.

Construir uma base sólida para o desenvolvimento saudável nos primeiros anos de vida é pré -requisito não apenas para o bem-estar individual, mas também para a produtividade econômica das sociedades. Evidências científicas demonstram que ambientes físicos e sociais desfavoráveis ameaçam o desenvolvimento humano e podem gerar prejuízos a longo prazo ${ }^{15}$. Sabendo-se que o desenvolvimento depende de fatores biológicos e ambientais deve-se considerar os tipos de estímulos que são oferecidos à criança.

Estudos identificaram consequências do tipo de estímulos oferecidos e dos papéis sociais esperados, oferecendo às crianças brinquedos $e$ propostas de atividades consideradas apropriadas para cada gênero ${ }^{16-21}$. Há evidências que esse tipo de estímulo direcionado pode ter influência no desempenho das áreas do DNPM ${ }^{22}$. Pesquisas mostram que diferenças neurológicas condicionam comportamentos diversos, observados desde o nascimento ${ }^{22,23}$. Van de Beek et al. ${ }^{24}$, corroboram com a relevância do binômio biologia-ambiente no DNPM das crianças (condições socioeconômicas, escolaridade dos pais, participação em atividades sociais entre outras).

Dentre os ambientes em que o DNPM vem sendo pesquisado, destacam-se ambulatórios ${ }^{25}$, instituições de acolhimento ${ }^{26}$ e escolas ${ }^{7,27}$. Neste último, predominam creches e pré-escolas por sua importância contextual nos primeiros anos de desenvolvimento da criança ${ }^{28,29}$. De acordo com Bronfenbrenner ${ }^{30}$, a escola é o segundo microssistema mais importante para a criança depois da família, e por isso seu reconhecimento como um ambiente importante para pesquisas.

O presente trabalho objetivou analisar o desenvolvimento neuropsicomotor de crianças matriculadas em Unidades de Educação Infantil (UEI) da rede pública do município de Belém, região Norte do país, a partir do seu desempenho no TTDD II, e assim estabelecer hipótese de 
relação entre tais resultados e a variável gênero da criança, bem como identificar os itens do teste em que essas crianças obtiveram mais falhas.

\section{Método}

Trata-se de um estudo de caráter exploratório, do tipo transversal, com abordagem quantitativa e descritiva dos dados. Estes foram coletados nas UEI de Belém, ligadas à Prefeitura Municipal e apresentam rotinas semelhantes, com pequenas variações de horário. Algumas unidades têm estrutura e recursos para a adequada realização das atividades, porém, muitas são improvisadas em casas alugadas, sem espaço para brincadeiras e atividades motoras amplas. As avaliações foram realizadas nas dependências internas das UEI, em geral no refeitório, ou em outro espaço que possuísse mesas e cadeiras adequadas à altura das crianças, seguindo todas as recomendações do manual do TTDD II.

As UEI foram distribuídas em oito Distritos Administrativos (DA): Distrito Administrativo de Belém (DABEL), do Guamá (DAGUA), do Benguí (DABEN), da Sacramenta (DASAC), do Entroncamento (DAENT), de Icoaraci (DAICO), Distrito de Mosqueiro (DAMOS) e de Outeiro (DAOUT). Os seis primeiros DA estão localizados na chamada Belém Continental (centro e periferia) e os dois últimos na Belém Insular (região das Ilhas).

Foi utilizado o processo de amostragem por conglomerado, com margem de erro do cálculo amostral de 5\% e o nível de confiança de $95 \%$. As UEI pesquisadas foram distribuídas segundo o número total em cada distrito, e de acordo com a quantidade de crianças pertencentes à faixa etária pesquisada. O estudo compreendeu de 19 UEI que foram selecionadas em um universo de 35 , conforme descrito na Tabela 1.

O banco de dados ficou composto por 318 crianças, após a exclusão de uma delas que não realizou todos os itens do teste. Foram selecionados ambos os gêneros, com idades entre $36 \mathrm{e}$ 48 meses, sem distúrbios na expressão da fala, alterações sensoriais, auditivas e/ou visuais e sequelas deixadas por comprometimento do SNC, além da apresentação de malformações, patologias osteoarticulares diagnosticadas ou qualquer outro tipo de doenças crônicas, graves e/ou debilitantes.

Os dados de caracterização dos sujeitos foram adquiridos com o Questionário de Características Biopsicossociais da Criança (QCBC),
Tabela 1. Distribuição das Crianças e das Unidades de Educação Infantil por Distrito Administrativo do Município de Belém.

\begin{tabular}{lcc}
\hline $\begin{array}{c}\text { Distrito } \\
\text { Administrativo } \\
(\mathbf{N}=\mathbf{8})\end{array}$ & $\begin{array}{c}\text { Número de UEI } \\
\text { Envolvidas na } \\
\text { Pesquisa }(\mathbf{N}=\mathbf{1 9})\end{array}$ & $\begin{array}{c}\text { Número de Crianças } \\
\text { Participantes da } \\
\text { Pesquisa }(\mathbf{N}=\mathbf{3 1 9})\end{array}$ \\
\hline DAGUA & 3 & 89 \\
DABEN & 3 & 59 \\
DASAC & 2 & 23 \\
DAENT & 3 & 46 \\
DAICO & 3 & 38 \\
DAMOS & 3 & 39 \\
DABEL & 1 & 7 \\
DAOUT & 1 & 18 \\
\hline
\end{tabular}

produzido especialmente para este estudo com base na literatura sobre fatores determinantes no desenvolvimento infantil. O questionário foi composto de 48 perguntas, sendo 19 abertas e 29 fechadas, direcionadas aos pais e distribuídas em torno dos eixos temáticos: (a) Identificação das crianças e pais (19 perguntas); (b) História pré, peri e pós-natal (6 perguntas); (c) Condições socioeconômicas e ambientais (20 perguntas).

Para obtenção dos dados sobre o desenvolvimento neuropsicomotor, utilizou-se o TTDD II $^{31}$, versão em espanhol, adaptada pelos pesquisadores, utilizando-se materiais do kit que acompanham o manual e outros necessários para a execução das tarefas (papel e lápis, mesa e cadeira infantil).

Esta pesquisa foi autorizada pela Secretaria Municipal de Educação (SEMEC) e aprovada pelo Comitê de Ética em Pesquisa com Seres Humanos do Núcleo de Medicina Tropical (NMT/ UFPA). Os procedimentos utilizados obedeceram às recomendações da Resolução no. 196/96 do Conselho Nacional de Saúde e da Comissão Nacional de Ética em Pesquisa, vigente à época.

Uma vez informada da autorização por escrito da SEMEC, a direção de cada UEI foi convidada a participar da pesquisa. Solicitou-se o acesso dos pesquisadores às suas dependências, às crianças e seus familiares, priorizando-se não atrapalhar a rotina da instituição ou causar qualquer desconforto.

O projeto foi apresentado nas visitas iniciais às UEI, havendo contato preliminar com as crianças, professores e ambiente da pesquisa. Nesta ocasião, pesquisadores se reuniam com professores e pais das crianças para leitura e assinatura do Termo de Consentimento Livre e Esclarecido 
(TCLE). O TTDD II foi aplicado às crianças na presença de seus pais e, quando inviável, de professoras indicadas pela instituição. O período de permanência dos pesquisadores nas UEI variou em função do número de crianças selecionadas e da disponibilidade da instituição para a coleta de dados, durando em média uma semana para cada instituição. A aplicação do teste acontecia individualmente com duração aproximada de 30 minutos. Durante o resto do tempo, os pesquisadores permaneciam na instituição interagindo com as crianças em outras atividades, visando sua inserção no ambiente e estreitamento da relação.

A pesquisadora responsável aplicou o TTDD II juntamente com dois auxiliares de pesquisa, previamente treinados, contando ainda com uma equipe de apoio. Cada criança foi testada em suas habilidades específicas esperadas dentro da sua faixa etária, com objetivo de verificar ritmos mais lentos ou mais rápidos de desenvolvimento. O desempenho das crianças em cada item foi avaliado e registrado, sendo seus dados anotados na Ficha de Registro dos Resultados. Nela, o termo Passou $(\mathrm{P})$ era registrado para indicar que a criança avaliada havia executado a tarefa solicitada com sucesso ou quando o seu cumprimento tivesse sido relatado pela mãe e/ou responsável familiar. Caso contrário, registrava-se Falhou (F).

Os dados coletados foram organizados em um banco por meio do software SPSS 20. A partir da natureza dos dados e do objetivo pretendido elaborou-se novo banco de dados, que reuniu informações sobre cada item avaliado e o percentual de $\mathrm{P}$ ou F. Foram geradas estatísticas descritivas e inferenciais. $\mathrm{O}$ teste de Kolmogorov-Smirnov foi aplicado para verificar a normalidade da distribuição amostral, o teste $t$ verificou as diferenças entre as médias das falhas por áreas do teste (variável dependente), segundo o gênero (variável independente); e o qui-quadrado verificou associação entre o número de falhas por item em relação ao gênero, todos considerando nível de significância de $5 \%$ ( $p$-valor $<0,05)$.

\section{Resultados}

A distribuição amostral foi considerada normal ( $p$-valor < 0,001). Das 318 crianças, 177 (56\%) eram meninos e 141 (44\%) meninas, com idade entre 36 e 48 meses, sendo $87 \%$ nascidos a termo. A maioria das famílias era de baixa renda, sendo que $31 \%$ recebia menos de um Salário Mínimo (SM), $65 \%$ entre um e três SM e apenas $4 \%$ possuíam renda superior a três SM. A maioria das mães declarou ter 12 anos ou mais de estudo $(40,4 \%)$, e realizar algum tipo de trabalho regular $(37,3 \%)$, enquanto os pais apresentavam de 9 a 11 anos de estudo (29,8\%), em sua maioria exercendo algum trabalho informal $(51,4 \%)$.

$O$ teste $t$ indicou diferença significativa entre os gêneros e o número de falhas obtidas pelas crianças em quase todas as áreas avaliadas pelo TTDD II, com exceção da área Motor Amplo. Em todas as áreas, médias mais altas foram associadas ao gênero feminino (Tabela 2) sugerindo melhor desempenho das meninas.

Buscou-se, ainda, relacionar os resultados com outras variáveis, além do gênero. Dentre as quais: escolaridade da mãe, escolaridade do pai, renda familiar e situação no nascimento (a termo ou pré-termo). Nenhuma delas, porém, mostrou associação estatisticamente significante com as falhas das crianças, sendo assim, elas foram omitidas dos resultados finais.

Uma análise mais detalhada demonstrou que o percentual de falha variou em razão do gênero. A Tabela 3 apresenta o desempenho das crianças em cada tarefa por área do desenvolvimento avaliada e por gênero.

Em resultados absolutos, observou-se indícios de desempenho inferior dos meninos em três das quatro áreas avaliadas e também na maioria dos itens citados $(\mathrm{N}=19)$. Apenas nos itens da área Motor Amplo as meninas tiverem um pior desempenho que os meninos.

$\mathrm{Na}$ Área da Linguagem, em que ambos os gêneros apresentaram piores desempenhos, com percentuais maiores de falhas para meninos, os itens observados com maiores diferenças estatísticas foram: "conhece 2 adjetivos", "conhece 4 ações" e "compreende 4 preposições". Nas áreas Pessoal Social e Motor Fino, em que meninos também tiveram pior desempenho, os itens mais falhos foram: "escova os dentes sem ajuda" e "indica a linha mais longa", respectivamente. $\mathrm{Na}$ área Motor Amplo, o item que apresentou maior diferença estatística foi "pula num pé só".

\section{Discussão}

\section{Desempenho por áreas}

Os resultados demonstram que meninas obtiveram desempenho melhor em três das áreas pesquisadas (Pessoal Social, Motor Fino e Linguagem), e em apenas uma das áreas (Motor Amplo) não houve diferença estatisticamente significativa entre gêneros. Esse dado corrobora 
Tabela 2. Desempenho de meninos e meninas por área avaliada pelo TTDD II (N = 318)

\begin{tabular}{|c|c|c|c|c|c|c|}
\hline & Gênero & $\mathbf{n}$ & Média & Desvio Padrão & $t$ & p-valor \\
\hline \multirow[t]{2}{*}{ Pessoal Social } & Feminino & 141 & 5,76 & 0,542 & \multirow{2}{*}{4,52} & \multirow{2}{*}{$<0,001^{\star}$} \\
\hline & Masculino & 177 & 5,41 & 0,842 & & \\
\hline \multirow[t]{2}{*}{ Motor Fino } & Feminino & 141 & 4,58 & 0,622 & \multirow{2}{*}{2,24} & \multirow{2}{*}{$0,020^{*}$} \\
\hline & Masculino & 177 & 4,41 & 0,718 & & \\
\hline \multirow[t]{2}{*}{ Linguagem } & Feminino & 141 & 7,38 & 1,547 & \multirow{2}{*}{2,63} & \multirow{2}{*}{$0,028^{*}$} \\
\hline & Masculino & 177 & 6,87 & 1,887 & & \\
\hline \multirow[t]{2}{*}{ Motor Amplo } & Feminino & 141 & 4,67 & 0,712 & \multirow{2}{*}{$-0,20$} & \multirow{2}{*}{0,586} \\
\hline & Masculino & 177 & 4,68 & 0,639 & & \\
\hline \multirow[t]{2}{*}{ Total } & Feminino & 141 & 22,49 & 2,236 & \multirow[t]{2}{*}{3,58} & \multirow[t]{2}{*}{0,003} \\
\hline & Masculino & 177 & 21,38 & 2,804 & & \\
\hline
\end{tabular}

Nota: Nível de significância de 5\%.

Tabela 3. Desempenho das crianças (passou, falhou) por gênero em relação a área.

\begin{tabular}{|c|c|c|c|c|c|}
\hline \multirow[b]{2}{*}{ Área } & \multirow{2}{*}{\multicolumn{2}{|c|}{ Item }} & \multirow[b]{2}{*}{$\begin{array}{c}\text { Não cumpriu a tarefa } \\
\text { Falhou }(\%)\end{array}$} & \multicolumn{2}{|c|}{ Gênero $\widehat{\partial} q$} \\
\hline & & & & $\chi^{2}$ & p-valor \\
\hline Pessoal & 1. & Veste-se sem ajuda & $\left(\circlearrowright^{\Uparrow} 0,6\right.$ e $\left.+1,4^{*}\right)$ & 0,612 & 0,434 \\
\hline \multirow[t]{5}{*}{ Social } & 2. & Escova os dentes com ajuda & $\left(\lesssim 7,3^{\star} \mathrm{e}+0,0\right)$ & 10,7 & $0,001^{\star}$ \\
\hline & 3. & Lava as mãos & $\left(\delta^{\star} 8,5^{*} \mathrm{e}+3,5\right)$ & 3,23 & 0,072 \\
\hline & 4. & Nomeia 1 amigo & $\left(\widehat{\partial} 25,4^{*} \mathrm{e}+9,5\right)$ & 3,56 & 0,081 \\
\hline & 5. & Veste a camisa & $\left(\circlearrowright^{\Uparrow} 16,9^{*}\right.$ e $\left.\subsetneq 9,9\right)$ & 3,24 & 0,072 \\
\hline & 6. & Prepara lanche simples & $(\widehat{\jmath} 0,00$ e $+0,00)$ & - & - \\
\hline \multirow[t]{5}{*}{ Motor Fino } & 1. & Imita a linha vertical & $\left(\widehat{o} 13,6^{*} \mathrm{e}+7,8\right)$ & 2,65 & 0,103 \\
\hline & 2. & Torre de 8 cubos & $\left(\jmath^{\star} 24,3^{*}+21,3\right)$ & 0,38 & 0,534 \\
\hline & 3. & Copia um circulo & $\left(\widehat{\partial} 13^{*} \mathrm{e}+8,5\right)$ & 1,61 & 0,204 \\
\hline & 4. & Copia uma cruz & 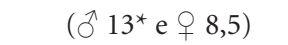 & 1,61 & 0,204 \\
\hline & 5. & Indica a linha mais longa & 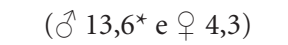 & 0,90 & $0,013^{*}$ \\
\hline \multirow[t]{9}{*}{ Linguagem } & 1. & Nomeia 4 figuras & $\left(\widehat{\jmath} 6,8^{*} \mathrm{e}\right.$ 車 3,5$)$ & 1,62 & 0,103 \\
\hline & 2. & Conhece 2 ações & $\left(\lesssim 6,8^{*} \mathrm{e} q 3,5\right)$ & 0,46 & 0,498 \\
\hline & 3. & Conhece 2 Adjetivos & $\left(\lesssim 37,3^{*}\right.$ e $\left.+25,5\right)$ & 4,97 & $0,026^{*}$ \\
\hline & 4. & Nomeia 1 cor & $\left(\widehat{\partial} 42,9^{*} \mathrm{e}+38,3\right)$ & 0,69 & 0,405 \\
\hline & 5. & Conta 1 bloco & $\left(\lesssim 33,3^{\star} \mathrm{e}+\right.$ O 29,1$)$ & 0,65 & 0,417 \\
\hline & 6. & Uso de 3 objetos & $\left(\delta^{\star} 40,7^{*} \mathrm{e}+\underline{+} 33,3\right)$ & 1,80 & 0,179 \\
\hline & 7. & Conhece 4 ações & $\left(\widehat{o} 15,8^{*} \mathrm{e}+8,2\right)$ & 3,88 & $0,049^{*}$ \\
\hline & 8. & Fala $100 \%$ inteligível & $\left(\delta_{1} 11,9^{*}\right.$ e $\left.q 9,9\right)$ & 0,30 & 0,584 \\
\hline & 9. & Compreende 4 preposições & $\left(\delta^{\star} 11,3^{*}\right.$ e $\left.q 4,3\right)$ & 5,18 & $0,023^{*}$ \\
\hline Motor & 1. & Pula & 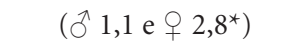 & 1,23 & 0,266 \\
\hline \multirow[t]{4}{*}{ Amplo } & 2. & Joga a bola de cima para baixo & $\left(\jmath^{\star} 6,2^{\star}\right.$ e $\left.+5,7\right)$ & 0,41 & 0,840 \\
\hline & 3. & Salto amplo & $\left(0^{\lambda} 10,9^{*} \mathrm{e}+7,3\right)$ & 1,15 & 0,283 \\
\hline & 4. & Equilibra-se em um pé só $2 \mathrm{seg}$. & $\left(\right.$ ふ 1,8 e $\left.q 5,7^{\star}\right)$ & 2,45 & 0,117 \\
\hline & 5. & Pula num pé só & $\left(\right.$ ふै $\left.2,8 \mathrm{e} q 8,5^{\star}\right)$ & 5,014 & $0,025^{*}$ \\
\hline
\end{tabular}

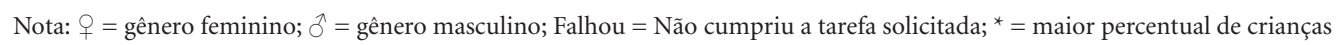
que não realizou a tarefa solicitada.

com o encontrado em estudo com pré-escolares realizado em Cuiabá, apontando que meninas apresentam desempenho superiores no TTDD ${ }^{10}$. Andrade e Negreiros ${ }^{32}$ também encontraram re- sultados de DNPM superiores em meninas nos dois primeiros anos de vida, provavelmente devido à mielinização mais rápida do córtex cerebral delas. Tal diferenciação também foi descrita uti- 
lizando-se a Alberta Infant Motor Scale, destacando-se novamente uma superioridade feminina no escore total e percentil aos 14 meses $^{4}$. Assim como meninos apresentaram maior tendência em suspeitas de atraso no desenvolvimento em áreas como motricidade ${ }^{33-35} \mathrm{e}$ linguagem ${ }^{36}$.

Há, porém, estudos que não encontraram relações significativas entre gênero e atraso no DNPM, nas áreas Pessoal Social e Linguagem ${ }^{7} \mathrm{e}$ desempenho motor ${ }^{37}$. Outros autores ${ }^{38}$ também não encontraram diferenças significativas comparando gênero com resultados globais do teste de desenvolvimento. Oliveira et al. ${ }^{39}$ mostraram que meninos têm competências motoras globais mais desenvolvidas do que meninas. Apenas um estudo sobre desempenho locomotor e controle de objetos na primeira infância de prematuros sugeriu prejuízo no desenvolvimento de meninas ${ }^{40}$.

Observa-se, assim, resultados de pesquisa bastante controversos. Autores divergem em tentar explicar as diferenças no desempenho de crianças de gêneros diferentes. Tais diferenças podem não estar diretamente associadas à variável gênero enquanto atributo de natureza biológica, mas à educação historicamente diferenciada em função das expectativas sociais de gênero e, portanto, da natureza das atividades ofertadas, como jogos e brincadeiras.

Assim, admite-se que os papeis sociais atribuídos para cada gênero se diferem desde o nascimento. Atividades como competição, contato físico e jogos de interdependência exigindo força, resistência e potência, com predomínio de ações envolvendo saltos e corridas, em espaços maiores, são características dos jogos e brincadeiras de meninos. Ao passo que meninas são estimuladas a atividades de natureza estética ou rítmica, com movimentos finos e mais controlados e em espaços menores ${ }^{37}$. Resultados semelhantes foram obtidos para a faixa etária entre sete e oito anos, quando se verificou entre os meninos maior preferência por brinquedos com componentes móveis e atividade motora em espaços amplos (por exemplo, bolas e carros); e entre meninas por brincadeiras mais estáticas e com comportamentos mais verbais do que motores, como por exemplo, brincar com bonecas ${ }^{17}$. Assim, as expectativas pessoais e sociais vivenciadas pela criança desde o seu nascimento poderiam direcionar seus comportamentos, de acordo com os papeis sociais esperados para cada gênero, e encaixando-se em estereótipos de gênero (atitudes, habilidades e traços de personalidade considerados apropriados para meninos e meninas segundo contexto sociocultural) $)^{41,42}$.
A partir do exposto, e considerando que o TTDD II é um teste construído a partir da execução de tarefas relacionadas a habilidades específicas de cada área que o compreende, pode-se sugerir que as diferenças de desempenho aqui apresentadas retratam o que é possível se observar na vida social. Se meninas são mais estimuladas a realizar atividades que envolvam predominantemente motricidade fina e habilidades de comunicação, em espaços geralmente mais restritos, então é compreensível que quando avaliadas nessas áreas apresentem um melhor desempenho que os meninos.

\section{Percentual de falhas por itens do teste}

Buscou-se identificar itens com mais falhas por gênero nas quatro áreas do teste. Como apresentado na Tabela 3, os itens mais falhos foram similares entre ambos. Para as meninas o percentual de tarefas não cumpridas com sucesso foi menor nas áreas: Pessoal Social, Motor Fino e Linguagem, as quais avaliam habilidades relacionadas à coordenação motora fina, interação $\mathrm{e}$ comunicação. Já na área Motor Amplo, que avalia habilidades como equilíbrio e coordenação motora global, ambos tiveram percentual pequeno, mas ligeiramente maior para as meninas.

Uma possível explicação para tal diversidade é que os gêneros diferenciam-se quanto às preferências no cotidiano. Meninas aparentam interessar-se mais por interações sociais e comunicação ${ }^{43}$, já os meninos tem maior habilidade motora para correr, saltar e deslizar ${ }^{39}$, o que justifica o desempenho diferenciado no teste. Gabbard ${ }^{44}$ corrobora com esse dado, afirmando que na primeira infância os meninos tendem a ter uma maior habilidade para correr e saltar, enquanto que as meninas têm maior facilidade com habilidades motoras finas e equilíbrio.

É provável que tais habilidades, ou sua ausência, sejam frutos de estímulos presentes no dia-a-dia das crianças. A influência do contexto e do tipo de tarefa vem sendo discutida e estudos demonstram que pais e agentes socializadores (professores, cuidadores, entre outros), frequentemente induzem as crianças a atividades consideradas adequadas para cada gênero, apoiando decisões, classificando brinquedos e brincadeiras, nas características sexuais biológicas ${ }^{16,37}$.

Para além das diferenças entre gêneros, os resultados mostram o expressivo número de itens em que as crianças falharam, indicando a necessidade de se discutir o desempenho delas de maneira geral. Vale destacar que o presente estudo 
não objetivava analisar as variáveis socioeconômicas impactantes no desempenho das crianças, mas como a triagem foi realizada na rede pública municipal de Belém, a população estudada ficou caracterizada quase que exclusivamente como de baixa renda. Por este motivo, não foi possível demonstrar por testes de regressão estatística uma possível influência dos fatores socioeconômicos sobre o DNPM das crianças estudadas.

Pesquisa anterior realizada nas mesmas instituições do presente estudo, relata que a seleção das crianças para ingresso nas UEI seria pelo grau de vulnerabilidade social de suas famílias, incluindo fatores socioeconômicos (especialmente renda dos cuidadores habituais). Segundo a autora, a ausência de saneamento básico e/ou água potável e a precariedade das condições de saúde da família e do ambiente imediato da criança, as exporiam a doenças, justificando a prioridade dos serviços públicos a tal população. Assim, um desempenho geral das crianças aquém do esperado para a sua faixa etária poderia ter relação direta com o baixo nível socioeconômico de suas famílias (renda, escolaridade, por exemplo) ${ }^{13}$.

A literatura aponta que baixos níveis socioeconômicos têm associação direta com a qualidade do desenvolvimento neuropsicomotor, apontando que além de fatores biológicos, as condições ambientais e socioeconômicas podem determinar atraso no $\mathrm{DNPM}^{45}$. Uma pesquisa buscando avaliar o desempenho motor global e habilidades motoras axiais e apendiculares de lactentes em contexto de creche encontrou 30\% de casos suspeitos de atraso motor global em pelo menos uma das avaliações realizadas. Tal resultado seria justificado pelo baixo nível de estimulação ambiental ou escassez de oportunidades para que o potencial de desenvolvimento fosse atingido ${ }^{46}$. Pesquisadores avaliaram o impacto da pobreza sobre o desenvolvimento de uma população de lactentes, descrevendo que cerca de $20 \%$ apresentavam frequência mais elevada de suspeita de atraso na comunicação receptiva e provinham de famílias que pertenciam ao quartil inferior do índice do nível socioeconômico. Segundo os autores, o desemprego materno e paterno reduziu, respectivamente, os escores de comunicação receptiva e cognição das crianças estudadas ${ }^{36}$.

Condições socioeconômicas desfavoráveis têm especial impacto quando vivenciadas na primeira infância. Pesquisadores identificaram prevalência de $17 \%$ de suspeita de atraso na área Motor Grosso e sua associação com fatores neonatais, familiares e de exposição à creche em crianças com até três anos, assistidas em creches públicas, com desvantagens em crianças até 24 meses em Habilidades de Locomoção. O risco de atraso no desempenho motor grosso aumentou 2,81 vezes para crianças de famílias com renda mensal de até R\$ 700,00; enquanto que o risco de atraso em Habilidades de Locomoção aumentou 4,63 vezes para filhos de pais com até oito anos de escolaridade ${ }^{47}$. Veleda et al. ${ }^{48}$, utilizando TTDDII, observaram que crianças de famílias com menor renda, inadequado índice de peso-idade e menos de seis consultas pré-natais, apresentavam maior suspeita de risco no desenvolvimento.

Dessa forma, observa-se a íntima relação entre ambiente e desenvolvimento infantil, gerando um ciclo em que vários fatores de risco se retroalimentam e têm impacto, por exemplo, pais com pouco estudo têm menos recursos para promover o desenvolvimento adequado dos filhos. Sabe-se que condições desfavoráveis podem estar associadas a atrasos no desenvolvimento infantil, impedindo o alcance do máximo potencial. Com o objetivo de analisar a relação entre desenvolvimento neuropsicomotor e ambiente familiar, uma pesquisa realizada em Belo Horizonte destaca que a estimulação pelo ambiente foi a variável de maior impacto no processo, demonstrando que a organização do ambiente físico pelos pais e a interação destes com seus filhos influencia diretamente o desenvolvimento infantil ${ }^{49}$.

Há estudos destacando que a inserção das crianças em UEI sem estrutura física e de capacitação profissional adequadas pode trazer risco ao seu desenvolvimento. Utilizar brinquedos inapropriados para a faixa etária, inadequação do local físico, falta de orientação pedagógica e de socialização extrafamiliar e baixa condição socioeconômica familiar, são fatores que podem impactar negativamente no desenvolvimento ${ }^{46}$. Uma pesquisa com crianças de diversas faixas etárias encontrou impactos negativos no desenvolvimento a partir dos três anos de idade, momento em que a proporção de alunos por cuidadores aumenta nas instituições ${ }^{7}$. Neste estudo, contudo, não se pode afirmar que estes aspectos tenham impactado os resultados encontrados, por não terem sido variáveis avaliadas. Logo, sugere-se que esses aspectos sejam futuramente considerados em pesquisas com objetivos, método e população semelhantes.

Além disso, é importante destacar que mesmo sem análises que permitam afirmar que o desempenho das crianças avaliadas no presente estudo tenha dependido do baixo nível socioeconômico de suas famílias, esta é uma variável relevante em se tratando de populações atendi- 
das pelos serviços públicos, como é o caso desta pesquisa. Entende-se que para uma análise mais abrangente e correlacional das variáveis com nível de influência significativa sobre as aquisições desenvolvimentais na população pesquisada, torna-se necessário investigar os estímulos oferecidos, as atividades realizadas e outras características do meio em que criança está crescendo. Nesse sentido, a ausência de informações acerca dos seus contextos de desenvolvimento caracteriza-se com uma limitação deste estudo.

\section{Considerações finais}

A análise dos resultados permitiu compreender aspectos importantes acerca do desempenho de meninos e meninas que frequentam as UEIs a partir do seu desempenho em um teste que avalia o desenvolvimento neuropsicomotor. As diferenças demonstram que meninas tiveram melhor desempenho que meninos, resultados que, conforme literatura, podem ter dependido mais dos estímulos recebidos no ambiente do que das diferenças biológicas.
Destaca-se a relevância de se compreender o desenvolvimento neuropsicomotor na primeira infância, em seus diversos aspectos, pois este é base para as aquisições futuras dessas crianças. Mesmo quando em situação de risco, com os estímulos adequados, as carências podem ser superadas e o desenvolvimento pode ocorrer satisfatoriamente. Em síntese, quanto melhor a qualidade da estimulação ambiental disponível para a criança, melhor será o seu desenvolvimento.

Por fim, entende-se que as preferências são construídas e a sociedade tende a contribuir para que as crianças pequenas sigam um padrão socialmente imposto do que considere certo ou errado, aceitável ou passível de rejeição e acabe optando por padrões de comportamento que limitam o seu desenvolvimento. Deste modo, aponta-se a necessidade de se promover políticas públicas que visem orientar pais e educadores que são os principais estimuladores nessa primeira etapa da vida, de modo que se conscientizem dessas diferenças e possam oferecer estímulos adequados ao desenvolvimento de todas as habilidades de suas crianças.

\section{Colaboradores}

M Lopes-Silva trabalhou na pesquisa, metodologia, concepção e redação final deste artigo; LIC Cavalcante na concepção e redação final; e S Heumann e TVR Lima na revisão, formatação e redação final. 


\section{Referências}

1. Engle PL, Fernald LCH, Alderman H, Behrman J, O'Gara C, Yousafzai A, Mello MC, Hidrobo M, Ulkuer N, Ertem I, Iltus S, The Global Child Development Steering Group. Strategies for reducing inequalities and improving developmental outcomes for young children in low-income and middle-income countries. Lancet 2011; 378(9799):1339-1353.

2. Martins TSA, Vilela RV, Pereira FV, Cária NZ, Faria HP. Implementação da avaliação do crescimento e do desenvolvimento neuropsicomotor em crianças menores de 5 anos na USF Grajaú na cidade de Brumadinho MG, pelo internato rural da UFMG. Revista Médica de Minas Gerais 2013; 23(1):27-32.

3. Guedes DZ, Primi R, Kopelman BI. BINS validation - Bayley neurodevelopmental screener in Brazilian preterm children under risk conditions. Infant Behav Dev 2011; 34(1):126-135.

4. Saccani R, Valentini NC. Curvas de referência da Escala Motora Infantil de Alberta: percentis para descrição clínica e acompanhamento do desempenho motor ao longo do tempo. Jornal de Pediatria 2012; 88(1):40-47.

5. Rosa Neto F. Manual de avaliação motora. Porto Alegre: Artmed; 2010.

6. Halpern R, Giugliani ERJ, Victora CG, Barros FC, Horta BL. Fatores de risco para suspeita de atraso no desenvolvimento neuropsicomotoraos 12 meses de vida. Jornal de pediatria 2000; 76(6):421-428.

7. Rezende MA, Beteli VC, Santos JLS. Follow-up of the child's motor abilities in day-care centers and preschools. Rev Lat Am Enfermagem 2005; 13(5):619-625.

8. Sigolo ARL, Aiello ALR. Análise de instrumentos para triagem do desenvolvimento infantil. Paidéia (Ribeirão Preto) 2011; 21(48):51-60.

9. Torquato JA, Paes JB, Bento MC, Saikai GMPN, Souto JN, Lima EAM, Abreu LC. Prevalência de atraso do desenvolvimento neuropsicomotor em pré-escolares. Revista brasileira de crescimento e desenvolvimento humano 2011; 21(2):259-268.

10. Souza SC, Leone C, Takano OA, Moratelli HB. Desenvolvimento de pré-escolares na educação infantil em Cuiabá, Mato Grosso, Brasil. Cad Saude Publica 2008; 24(8):1917-1926.

11. Carneiro JM, Brito APB, Santos MEA. Avaliação Do Desenvolvimento De Crianças De Uma Creche Através Da Escala Denver II. Revista Mineira de Enfermagem 2011; 15(2):174-180.

12. Magalhães LC, Fonseca KL, Martins LDTB, Dornelas LF. Desempenho de crianças pré-termo com muito baixo peso e extremo baixo peso segundo o teste Denver-II. Revista Brasileira de Saúde Materno Infantil 2011; 11(4):445-453.

13. Guerreiro TBF. Desenvolvimento neuropsicomotor de crianças de Belém: associação com características pessoais e variáveis do seu ambiente ecológico [tese]. Belém: Universidade Federal do Pará; 2013.

14. Costa EF. Desenvolvimento Linguístico de Crianças de Belém: Associação com características pessoais e ambientais [tese]. Belém: Universidade Federal do Pará; 2014.

15. Shonkoff JP, Richter L, Gaag JV, Bhutta ZA. Development An Integrated Scientific Framework for Child Survival and Early Childhood. Pediatrics 2012; 129(2):e460-472.
16. AltmannH, Ayoub E, Amaral SCF. Gênero na prática docente em educação física: meninas não gostam de suar, meninos são habilidosos ao jogar? Rev Est Fem 2011; 19(2):491-501.

17. Carvalhal MIM, Vasconcelos-Raposo J. Diferenças entre gêneros nas habilidades: correr, saltar, lançar e pontapear. Motricidade 2007; 3(3):44-56.

18. Finco D. Relações de gênero nas brincadeiras de meninos e meninas na Educação Infantil. Pro-Posições: Dossiê Educação Infantil e Gênero 2003; 14(42):89-102.

19. Vianna C, Finco D. Meninas e meninos na Educação Infantil: uma questão de gênero e poder. Cad. Pagu 2009; (33):265-283.

20. Menezes AB, Brito RCS, Henriques AL. Relação entre Gênero e Orientação Sexual a partir da Perspectiva Evolucionista. Psicologia: Teoria e Pesquisa 2010; 26(2):245-252.

21. Menezes AB, Brito RCS. Diferenças de gênero na preferência de pares e brincadeiras de crianças. Psicologia Reflexão e Crítica 2013; 26(1):193-201.

22. Alexander GM, Wilcox T, Woods R. Sex differences in infants' visual interest in toys. Arch Sex Behav 2009; 38(3):427-433.

23. Hansen J, Macarini SM, Martins GDF, Wanderlind FH, Vieira ML. O brincar e suas implicações para o desenvolvimento infantil a partir da Psicologia Evolucionista. Revista Brasileira de Crescimento e Desenvolvimento Humano 2007; 17(2):133-143.

24. Van de Beek C, Van Goozen SHM, Buitelaar JK, Cohen-Kettenis PT. Prenatal sex hormones (maternal and amniotic fluid) and gender related play behavior in 13-month-old infants. Arch Sex Behav 2009; 38(1):615.

25. Moraes MW, Weber APR, Santos MCO, Almeida FA. Denver II: evaluation of the development of children treated in the outpatient clinic of Project Einstein in the Community of Paraisópolis. Einstein 2010; 8(21):149-153.

26. Lima AKP, Lima AO. Neuropsychomotor develompent profile and family aspects of institutionalized children living in Recife. Revista CES Psicología 2012; 5(1):1125.

27. Santos AM, Neto FR, Pimenta RA. Avaliação das habilidades motoras de crianças participantes de projetos sociais/esportivos. Motricidade 2013; 9(2):50-60.

28. Stich HL, Baune BT, Caniato RN, Mikolajczyk RT, Krämer A. Individual development of preschool children-prevalences and determinants of delays in Germany: a cross-sectional study in Southern Bavaria. BMC Pediatrics 2012; 12:188.

29. Bernal R, Fernández C. Subsidized childcare and child development in Colombia: Effects of Hogares Comunitarios de Bien estar as a function of timing and length of exposure. Soc Sci Med 2013; 97:241-249.

30. Bronfenbrenner U. A ecologia do Desenvolvimento Humano: Experimentos Naturais e planejados. Porto Alegre: Artes médicas; 1996.

31. Frankenburg WK, Dodds J, Archer P, Shapiro H, Bresnick B. The Denver II: a major revision and restandardization of the Denver Developmental Screening Test. Pediatrics 1992; 89(1):91-97 
32. Andrade JL, Negreiros MM. Suspeita de atraso no desenvolvimento neuropsicomotor em crianças menores de um ano atendidas em uma unidade de saúde da família de rio branco (acre). Revista APS 2013; 16(1):6065.

33. Braga AKP, Rodovalho JC, Formiga CKMR. Evolução do crescimento e desenvolvimento neuropsicomotor de crianças pré-escolares de zero a dois anos do município de Goiânia (GO). Revista brasileira de crescimento e desenvolvimento humano 2011.21(2):230-239.

34. Brito CML, Vieira GO, Costa MCO, Oliveira NS. Desenvolvimento neuropsicomotor: o teste de Denver na triagem dos atrasos cognitivos e neuromotores de pré -escolares. Cad Saude Publica 2011; 27(7):1403-1414.

35. Fernandes LV, Goulart AL, Santos AMN, Barros MCM, Guerra CC, Kopelman BI. Neurodevelopmental assessment of very low birth weight preterm infants at corrected age of 18-24 months by Bayley III scales. Jornal de Pediatria 2012; 88(6):471-478.

36. Paiva GS, Lima ACVMS, Lima MC, Eickmann SH. O efeito da pobreza no escore de triagem do desenvolvimento de lactentes. Sao Paulo Med. J. 2010; 128(5):276283.

37. Venturella CB, ZanandreaG, Saccani R, Valentini NC. Desenvolvimento motor de crianças entre 0 e 18 meses de idade: Diferenças entre os sexos. Motricidade 2010; 9(2):3-12.

38. Biscegli TS, Polis LB, Santos LM, Vicentin M. Suspeita de atraso no desenvolvimento neuropsicomotor em crianças menores de um ano atendidas em uma unidade de saúde da familia de Rio Branco. Revista Paulista de Pediatria 2007; 25(4):337-342.

39. Oliveira DS, Oliveira LS, Cattuzzo MT. A influência do gênero e idade no desempenho das habilidades locomotoras de crianças de primeira infância. Revista brasileira de educação física e esporte 2013; 27(4):647-655.

40. Campos CMC, Soares MMA, Cattuzzo MT. O efeito da prematuridade em habilidades locomotoras e de controle de objetos de crianças de primeira infância. Motriz: rev. educ. fis. 2013; 19(1):22-33.

41. Papalia DE, Olds SW, Feldman, RT. Desenvolvimento Humano. Porto Alegre: Art Med; 2010.

42. Haywood KM, Getchell N. Desenvolvimento Motor ao longo da vida. Porto Alegre: Art Med; 2010.

43. Garcia C. Gender differences in young children's interactions when learning fundamental motor skills. Res $Q$ Exerc Sport 1994; 65(3):213-225.
44. Gabbard C. Lifelong motor development. $4^{\text {th }}$ ed. San Francisco: Pearson; 2004.

45. Saccani R, Brizola E, Giordani AP, Bach S, Resende TL, Almeida CS. Avaliação do desenvolvimento neuropsicomotor em crianças de um 89 bairro da periferia de Porto Alegre. Scientia Medica 2007; 17(3):130-137.

46. Souza CT, Santos DCC, Tolocka RE, Baltier L, Gibim NC, Habechian FAP. Avaliação do desempenho motor global e em habilidades motoras axiais e apendiculares de lactentes frequentadores de creche. Revista Brasileira de Fisiotererapia 2010; 14(4):309-315.

47. Santos DCC, Tolocka RE, Carvalho J, Heringer LRC, Almeida CM, Miquelote AF. Desempenho motor grosso e sua associação com fatores neonatais, familiares e de exposição à creche em crianças até três anos de idade. Revista Brasileira Fisioterapia 2009; 13(2):173-179.

48. Veleda AA, Soares MCF, Cézar-Vaz MR. Fatores associados ao atraso no desenvolvimento em crianças, Rio Grande, Rio Grande do Sul, Brasil. Revista Gaúcha de Enfermagem 2011; 32(1):79-85.

49. Guimarães AF, Carvalho DV, Machado NAA, Baptista RAN, Lemos SMA. Risco de atraso no desenvolvimento de crianças de dois a 24 meses e sua associação com a qualidade do estímulo familiar. Revista Paulista de Pediatria 2013; 31(4):452-458.

Artigo apresentado em 02/12/2015

Aprovado em 02/09/2016

Versão final apresentada em 04/09/2016 